\title{
Urban Air Mobility Airspace Integration Concepts and Considerations
}

\author{
David P. Thipphavong ${ }^{1}$, Rafael D. Apaza, Bryan E. Barmore, Vernol Battiste, Barbara K. Burian, Quang V. Dao, \\ Michael S. Feary, Susie Go, Kenneth H. Goodrich, Jeffrey R. Homola, Husni R. Idris, Parimal H. Kopardekar, Joel \\ B. Lachter, Natasha A. Neogi, Hok K. Ng, Rosa M. Oseguera-Lohr, Michael D. Patterson, Savita A. Verma \\ National Aeronautics and Space Administration
}

\begin{abstract}
Urban Air Mobility - defined as safe and efficient air traffic operations in a metropolitan area for manned aircraft and unmanned aircraft systems - is being researched and developed by industry, academia, and government. Significant resources have been invested toward cultivating an ecosystem for Urban Air Mobility that includes manufacturers of electric vertical takeoff and landing aircraft, builders of takeoff and landing areas, and researchers of the airspace integration concepts, technologies, and procedures needed to conduct Urban Air Mobility operations safely and efficiently alongside other airspace users. This paper provides high-level descriptions of both emergent and early expanded operational concepts for Urban Air Mobility that NASA is developing. The scope of this work is defined in terms of missions, aircraft, airspace, and hazards. Past and current Urban Air Mobility operations are also reviewed, and the considerations for the data exchange architecture and communication, navigation, and surveillance requirements are also discussed. This paper will serve as a starting point to develop a framework for NASA's Urban Air Mobility airspace integration research and development efforts with partners and stakeholders that could include fast-time simulations, human-in-the-loop simulations, and flight demonstrations.
\end{abstract}

\section{Introduction}

VIATION technologies and concepts (e.g., for distributed electric propulsion [1] and service-oriented paradigm and architecture [2]) have reached a level of maturity to enable Urban Air Mobility (UAM) using quiet and efficient manned and unmanned vehicles to conduct on-demand and scheduled operations. Types of operations could include emergency medical evacuations, rescue operations, humanitarian missions, news gathering, ground traffic flow assessment, weather monitoring, package delivery, and passenger transport. With regard to the latter, a recent study evaluated potential takeoff and landing areas in the San Francisco Bay Area and concluded that the door-to-door trip time savings resulting from UAM could be significant for trips greater than 15 miles [3]. A number of technical challenges must be overcome to achieve mature UAM operations at higher tempo and higher density than can be accommodated by the current air traffic control (ATC) system.

Significant progress has already been made toward defining what future UAM operations could look like. For instance, Uber Elevate published a white paper describing their vision for an air taxi service [4]. It includes an analysis of the feasibility of using electric vertical takeoff and landing (eVTOL) vehicles for air taxi services, the economics of the air taxi market, and the ground infrastructure (e.g., vertiports, charging systems) required for air taxi operations. It also discusses several crucial airspace integration challenges for UAM, such as efficient sequencing and scheduling of aircraft into and out of vertiports and interoperability between vehicles. However, the Uber Elevate white paper focuses on air taxi operations and does not describe the broader range of missions, aircraft types, airspace, and hazards in metropolitan areas that this paper on UAM airspace integration does. Besides Uber Elevate, several other companies are also pursuing concepts for air taxi services, including Airbus in São Paulo, Brazil [5], Kitty Hawk in New Zealand [6], and Volocopter in Dubai, United Arab Emirates [7].

In recent years, a number of studies have been conducted on the subject of On-Demand Mobility (ODM) - air traffic operations between any origin and any destination [8] without the delays associated with scheduled service as in traditional commercial aviation. These include research efforts on the ODM vision [8], concepts [9], barriers [10], vehicles [11], demand forecasting [12], and impact on the National Airspace System (NAS) [13]. UAM is the subset

\footnotetext{
${ }^{1}$ Acting Sub-Project Manager for Initial Urban Air Mobility Operations Integration, Mail Stop 210-10, Moffett Field,
} CA 94035, AIAA Senior Member. 
of ODM that is focused on air traffic operations in metropolitan areas with aircraft capable of seating a small number of passengers or equivalent volume of goods flying trips of about 100 nautical miles (nmi) or less.

The technologies and procedures required for ODM were investigated in a NASA study [14] that covered the range of the airspace integration problem, including mission planning, separation from hazards (e.g., terrain, obstacles, other aircraft), contingency management, demand-capacity balancing, traffic flow management, as well as sequencing, scheduling, and spacing. A similar spectrum of topics will be covered in this complementary paper on UAM airspace integration. This paper also describes at a high level NASA's initial airspace integration concepts for both emergent and early expanded UAM operations. It also serves as a framework for NASA's UAM airspace integration research and development efforts with partners and stakeholders.

The remainder of this paper is organized as follows. Section II reviews past and current UAM operations. Section III presents an overview of UAM, including the goals, principles, barriers, and benefits. This section also discusses the competing considerations that need to be taken into account and balanced for UAM operations, as well as the requirements for communication, navigation, and surveillance. Section IV defines the scope of the concepts with regard to missions, aircraft, airspace, and hazards. Section V describes at a high level NASA's initial airspace integration concepts for both emergent and early expanded UAM operations. Section VI discusses NASA's plan to develop and refine the initial airspace integration concepts for both emergent and early expanded UAM operations that are described in this paper, as well as develop a concept for high-tempo, high-density mature operations, with the broader UAM community. This section also describes one framework currently being explored by NASA's Air Traffic Management-eXploration (ATM-X) project [15] for managing UAM operations. Lastly, the contributions of this paper toward defining UAM airspace integration concepts are summarized.

\section{Background}

The desire to conduct high-tempo, high-density air traffic operations within metropolitan areas has persisted for many decades. This section describes past and current UAM operations in the United States and other countries.

\section{A. Past Operations}

Commercial UAM operations have occurred in the United States since at least the 1940s. For example, from 1947 to 1971, Los Angeles Airways used helicopters to transport people and mail between dozens of locations in the Los Angeles basin, including Disneyland and Los Angeles International Airport. Tragically, the company experienced two accidents - both of which occurred in 1968 and were caused by mechanical failures - that killed several dozens of passengers and crew members [16]. After those incidents, the company's helicopters were grounded, and the resulting financial difficulties led Los Angeles Airways to cease operations a few years later.

During this same era, from 1949 to 1979, New York Airways primarily used helicopters to fly people between heliports in Manhattan and airports in the New York area such as LaGuardia, JFK, and Newark. However, New York Airways also stopped flying due to several incidents of mechanical failures that caused the deaths and injuries of dozens of passengers, crew members, and bystanders at heliports; during one tragic incident in 1977, parts of a broken helicopter blade fell from the helipad on the roof of the Pan Am Building to the ground, killing one pedestrian and injured another [17].

\section{B. Current Operations}

Although New York Airways ceased operations in 1979, there is still a demand for commercial UAM operations to avoid transportation congestion on the ground. In the United States, there are companies currently providing these services. For example, BLADE Bounce is an on-demand helicopter transfer service between BLADE helipad locations and New York area airports - both commercial and private. Customers can charter their own helicopter or book a seat on one of several scheduled flights each day. However, even at these low numbers of operations, the noise generated by BLADE helicopters has been significant enough to compel communities, such as the town of East Hampton, to take legal action to restrict the number of flights and when they could occur [18].

Outside of the United States, there is also consumer demand for UAM operations in gridlocked metropolitan areas such as São Paulo, Brazil, where Airbus is operating its Voom on-demand helicopter service [5]. In addition, Kitty Hawk announced testing of its Cora (formerly Zee Aero) autonomous planes in New Zealand with its operator Zephyr Networks as part of an official certification process [6]. Also, Volocopter has conducted testing in Dubai, United Arab Emirates toward developing an air taxi service there [7].

In addition to passenger-carrying flights, UAM also includes aircraft operations for tasks in metropolitan areas such as public safety, medical evacuations and rescue, news gathering, ground traffic assessment, weather monitoring, and package delivery. Outside of the United States, Amazon has conducted live flight tests of small UAS (sUAS) 
delivering orders to customers in England [19]. In addition, Zipline is using sUAS to transport blood, vaccines, and other medical supplies to remote clinics in Rwanda [20]. In the United States, NASA, the FAA, industry, and academia have been actively working toward the development and demonstration of a UAS Traffic Management (UTM) system for sUAS operations in low-altitude airspace [2]. Evaluations for sparsely-populated and moderately-populated areas have been conducted in the United States in recent years [21, 22], and testing for higher-density urban areas (UTM Technology Capability Level 4) is scheduled for the near future [2]. The extent to which the UTM architecture, paradigm, and principles could be applied to UAM is a crucial area of future research. It is expected that sUAS will need to conform to UAM requirements to operate in higher-altitude airspace. It will be critical to properly manage the interaction of sUAS and UAM vehicles with humans on board, especially around vertiports.

\section{Overview}

In collaboration with partners and stakeholders over the next few years, NASA will develop detailed concepts of operations for UAM airspace integration at different stages of operational maturity. This paper only provides highlevel concept descriptions for both emergent and early expanded UAM operations; a separate effort is planned to define concepts for mature UAM operations.

- Emergent UAM operations: Characterized by low-tempo, low-density flights along a small set of fixed routes between a few takeoff and landing areas

- Early expanded UAM operations: Characterized by higher-tempo, higher-density flights in a small network of vertiports feeding a common hub location and managed by UAM operator and third-party services

- Mature UAM operations: Characterized by high-tempo, high-density flights in a network with multiple hub locations, potentially with orders-of-magnitude more vehicles and operations in an area than are currently supported in the NAS

It is expected that the earliest UAM operations of the future will be for demonstration purposes and will be required to comply with current airspace rules and regulations. It is anticipated that they will be very similar to (if not the same as) current VFR operations. Furthermore, it is expected that these initial UAM flights will be conducted by human pilots on board the UAM aircraft at all times and will have ATC providing services as in current operations. However, a number of organizations are pursuing UAM concepts that span the range of possible roles and responsibilities for humans and autonomous systems. For instance, some UAM aircraft manufacturers are working on developing vehicles that would not require a human pilot on board. These aircraft would instead be piloted remotely by some combination of humans on the ground, autonomous systems on the ground, and/or autonomous systems in the air on board the aircraft.

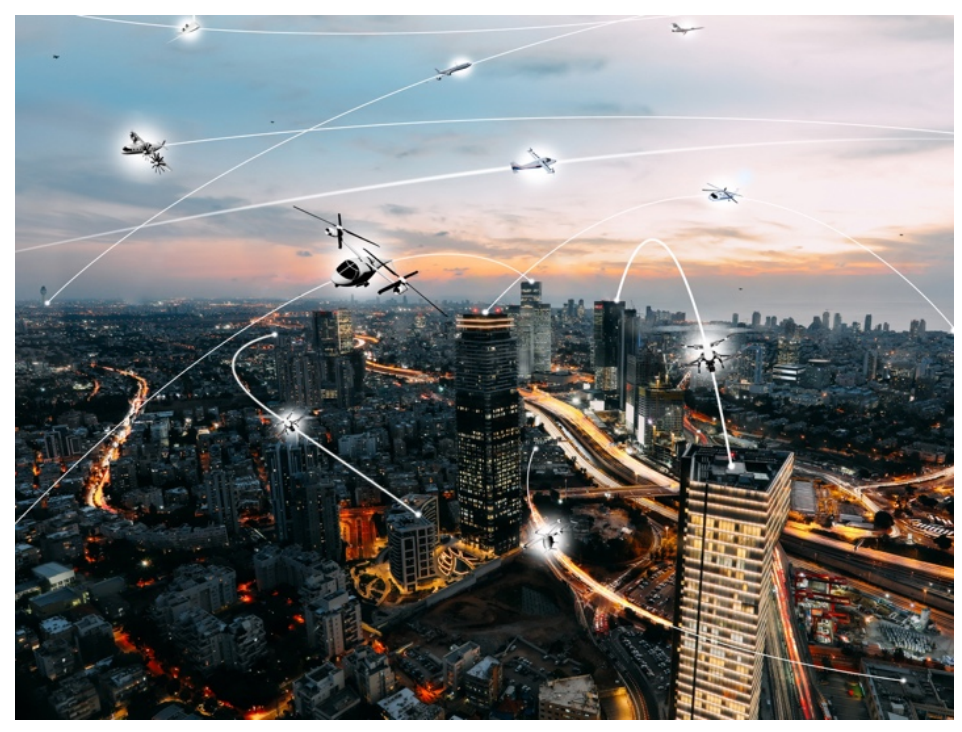

Fig. 1 Conceptual illustration of Urban Air Mobility operations. 
Beyond pilots, the roles and responsibilities of ATC may also need to be changed from current operations, such as transitioning from actively managing flights to monitoring. The roles and responsibilities of humans and autonomous systems in UAM could vary considerably for different concepts. This section details a set of principles for UAM airspace integration and discusses the barriers, benefits, and competing considerations that need to be taken into account and balanced when developing UAM airspace integration concepts, from low-tempo, low-density emergent UAM operations through high-tempo, high-density mature UAM operations.

\section{A. Goals and Principles}

Integration of UAM aircraft and operations into the airspace system will require them to be safe, efficient, and predictable, with minimal impact on existing airspace operations. This does not necessarily mean that UAM flights will be required to completely avoid other flights; the full breadth of aircraft and operations must be supported equitably. NASA's development of UAM airspace integration concepts, technologies, and procedures will be governed by the following set of guiding principles informed by prior work on ODM tenets [14]. This set of principles could evolve as NASA learns with and collaborates with the UAM community and the broader aviation community.

1. UAM should require minimal additional ATC infrastructure (e.g., radar systems, controller positions) and minimal changes to FAA automation systems used for ATC.

2. UAM should impose minimal additional workload on controllers beyond their current duties for existing airspace users.

3. UAM should impose minimal additional requirements or burdens on existing airspace users beyond equitable access to airspace resources.

4. UAM will meet the regulatory requirements for vehicle-level and system-level safety and security, such as timely and assured data exchange and the elimination of single points of failure and common failure triggers.

5. UAM will be resilient to a wide range of disruptions, from weather and localized sub-system failures (e.g., a single vehicle or software tool) to widespread disruptions (e.g., GPS failure).

6. UAM will economically scale to high-demand operations with minimal fixed costs.

7. UAM will support user flexibility and decision making to the greatest extent possible and enforce airspace structure and prescriptive procedures only as necessary to meet the above principles.

\section{B. Barriers}

A number of barriers must be overcome for UAM operations to be integrated safely and efficiently into the airspace system and for operations to be conducted at high tempo and high density with significantly more vehicles and operations in an area than are currently supported in the NAS. The barriers that are more closely associated with UAM vehicles include ride quality, lifecycle emissions, ease of certification in terms of both time and cost, auditory and visual noise in terms of annoyance perceived by the community on the ground, affordability in terms of operating cost, safety in terms of casualties and property damage, and efficiency in terms of energy usage.

A number of UAM airspace integration barriers must be tackled in addition to these UAM vehicle-related barriers. For instance, establishing safety will require the development of technologies and procedures to ensure separation from terrain, urban obstacles, and other aircraft. Furthermore, tools and methods to sequence, schedule, and space UAM aircraft at constrained resources, such as takeoff and landing areas (e.g., vertiports), are also needed for efficient operations. Overcoming the safety and efficiency barriers for airspace integration will require UAM vehicles and systems to be interoperable with each other as well as those of existing airspace users. At a minimum, standards must be developed for the data exchange architecture and for communication, navigation, and surveillance (CNS).

\section{Considerations to be Balanced}

The experiences of past and current UAM operations has shown that a number of competing considerations must be taken into consideration and balanced as the UAM community proceeds toward high-density, high-tempo mature UAM operations in the future. Many of these considerations are the same as for the integration of other new entrants to the airspace, such as sUAS [2]:

- Economic value: The aerial transport of people and goods in metropolitan areas for commercial, public safety, and personal use potentially has high economic benefit to the nation. Significant investments have been made by UAM infrastructure developers, vehicle manufacturers, and prospective operators in pursuit of these benefits. ATM concepts, technologies, and procedures to integrate UAM aircraft and operations into the airspace system are needed to enable and facilitate the expeditious capture of these economic benefits. 
- Security: Even as UAM enhances the overall mobility of people and goods in a metropolitan area, the ATM system must carefully and thoroughly identify high-value areas and assets, as well as set up appropriate controls to protect them. Airspace controls could include defining restricted airspace and prohibited airspace areas around these locations. Ground controls could include security and screening at takeoff and landing areas. ATM system controls include cybersecurity to assure safe and reliable exchange of vehicle data, prognostics data, critical navigation data, command and control, weather data, and other information.

- Safe airspace operations: UAM aircraft may need to operate alongside the full range of manned and unmanned aircraft currently in the airspace that have a wide range of equipage and capabilities operating under either Instrument Flight Rules (IFR) or Visual Flight Rules (VFR). Furthermore, unlike traditional aviation, which by design typically spends the majority of flight time over sparsely-populated areas, UAM operations by definition will generally occur over metropolitan areas that are densely populated in terms of both people and property. As such, a UAM mishap almost certainly would result in substantial damage. Thus, it is crucial that UAM concepts, technologies, and procedures be designed with safety in mind from the start.

- Community acceptance: As with any new entrant to the airspace, UAM aircraft and operations must be designed in a way to earn acceptance by the general public and also by the broader aviation community. Concerns about potential privacy violations, auditory and visual disturbances, safety risks, and affordability are some of the biggest factors that must be mitigated. The lessons learned from recent efforts to integrate both sUAS and large UAS into the airspace system can guide the UAM community.

The potential economic value of UAM operations is extensive, but the desire to operate anywhere anytime will need to be balanced against security, safe airspace operations, and community acceptance. UAM operations will not be allowed to occur at all times or at all locations when and where the security risks, safety risks, and other detrimental impacts to people and property in the air and on the ground are deemed to be too high. The threshold at which UAM operations will not be permitted is outside of the scope of this paper; this is something that will need to be determined by individual communities and the government at the local, state, and national levels.

\section{Transportation Benefits}

Besides the economic value of UAM, there are also potential benefits to the overall transportation system. The transportation benefits of UAM operations were investigated and described in detail in prior research efforts [3, 4]. A brief summary is presented in this section to provide context for this paper on UAM airspace integration concepts and considerations.

The most fundamental motivation for UAM is transporting people and goods around metropolitan areas at doorto-door speeds greater than and in a manner complementary to current air and ground transportation capabilities. UAM was found to have the potential for door-to-door trip times that are half or less than ground transportation, even when accounting for ground transportation times to/from takeoff and landing areas and intermodal transition penalties [3]. The potential time savings of UAM could be on the order of tens of minutes, which could be lost if the ATM system for UAM does not carefully manage and avoid potential sources of congestion and delay (e.g., bad weather).

In addition to reducing average trip times, UAM also has the potential to reduce uncertainty in travel times relative to ground transportation in highly-congested cities. In many major metropolitan areas, the "planning time index" (PTI) - an indication of the travel time allocation needed to assure a 95\% probability of arriving prior to a designated time - is often two to three times greater than the free-flow travel time [23]. Since UAM is a node-based transportation system of takeoff and landing areas rather than a path-based transportation system like ground surface roads [3], the former may be more resilient than the latter to disruptions, such as the closing of a critical roadway or bridge, that contribute to high PTI values for the latter. Furthermore, total transportation system capacity is expected to increase because UAM generally operates in parallel with ground transportation and does not compete for the same space.

Another potential benefit is that UAM may require relatively little ground infrastructure and can potentially be implemented more quickly and at lower cost compared to other ground-based modes. Instead of extensive roads, overpasses, bridges, tracks, and/or right-of-ways, the primary physical infrastructure needed by UAM are vertiports that have relatively small footprints. This is expected to result in smaller capital expenditures to integrate UAM into metropolitan areas and more rapid expansion of connectivity between existing areas or into new areas, compared to ground-based modes. On the other hand, if most UAM aircraft are electrically-powered [4], UAM could add significant electrical infrastructure requirements for electrical power generation and transmission. Fortunately, it is expected that the electrical infrastructure will be expanded anyway to support the anticipated growth in the number of ground vehicles with electric powertrains. 


\section{E. Communications, Navigation, and Surveillance}

To capture the potential economic value and transportation benefits of UAM discussed in previous sections, the requirements and infrastructure for CNS in UAM must be researched and developed. It is anticipated that some of the CNS services used in the NAS today (e.g., GPS) could be used without any changes. Technologies like Automatic Dependent Surveillance (ADS) and the location of ground receivers may require expansion to cover additional airspace requirements. Architecting CNS services for UAM is dependent on airspace configuration, route structure, vehicle capabilities, and safety requirements. In addition, cybersecurity is and will continue to be a challenge. Authentication mechanisms beyond today's system security must be developed, and methods to detect intrusions, data leaks, and other anomalies will be necessary.

Bonding CNS services and enabling seamless dissemination of information over diverse sub-networks will be a robust intranet backbone. Current ground-ground networks are using Internet Protocol (IP) networks to enable System Wide Information Management (SWIM) data distribution, weather dissemination, decision collaboration, and more. Efforts are underway by national and international standardization and regulatory organizations to standardize and enable air-ground IP to the cockpit [24]. IP Mobility, address configuration, domain naming, and multi-link utilization are a few of the different profiles currently being developed [25]. Using new Internet Protocol version 6 (IPv6) features and improvements, air-ground IP will seamlessly integrate with ground-ground networks to provide end-to-end connectivity.

\section{Communications}

Voice communications will always be available as a means of backup communications. Satellite and terrestrial link technologies with different service classes, quality of services, availability and price points will be accessible to users. The types of missions, business needs, geography, and equipment requirements will determine the type of link technology employed. To meet the needs of UAM early entrants, analog voice communications and existing data communications systems will be leveraged for safety-critical exchanges. The use of IP will enable data classification, segregation, and prioritization and will make UAM airspace integration possible. Communications systems will be required to securely and reliably transport vehicle data, prognostics data, critical navigation data, command and control, weather data, and other information.

\section{Navigation}

It is expected that the navigation services for UAM operations will use a combination of currently available and new technologies to guide aircraft from takeoff through landing. It is anticipated that UAM aircraft will navigate using GPS and ground-based navigational aids, such as Very High Frequency Omni-Directional Range (VOR)/Distance Measuring Equipment. As aircraft densities increase, GPS users could be required to use Wide Area Augmentation System to increase position precision. Navigation systems will have to be redundant to meet safety requirements. During arrival into takeoff and landing areas, UAM aircraft may need precision guidance to safely guide them through landing. This is particularly critical in poor-visibility situations, high-wind conditions, and densely populated areas, especially those with multiple buildings and other fixed obstacles in the vicinity. A viable navigation option in poorvisibility conditions could include the use of synthetic vision and terrain to provide the pilot with augmented reality to navigate in conditions that would not be possible otherwise.

\section{Surveillance}

Current surveillance technologies are classified into cooperative and non-cooperative. Cooperative surveillance requires aircraft equipment to respond to interrogations or automatically report position. Non-cooperative systems depend on electromagnetic reflections to detect target location. UAM aircraft can be tracked using available cooperative ADS-B terrestrial or space-based surveillance. ADS-B is a broadcast technology approved to operate in the $1090 \mathrm{MHz}$ and $978 \mathrm{MHz}$ frequency bands. There are valid concerns that the $1090 \mathrm{MHz}$ band is becoming saturated. Consequently, ADS-B broadcasts in the $978 \mathrm{MHz}$ band are a viable option for UAM. An emerging concept that is an additional option for cooperative surveillance is ADS-IP, where position information is sent using IPv6 networks over data link systems. UAM can also benefit from Wide Area Multilateration, which uses transponder replies to triangulate and derive position, similar to airport surface surveillance Multilateration.

Non-cooperative surveillance for UAM operations is a challenge. High-resolution video cameras utilizing image recognition and deployed at strategic locations can detect vehicles and other objects in the airspace during daytime. For night operations, the camera system can utilize infrared detection to track vehicles. Passive radar is a viable solution for target detection in adverse weather conditions such as rain and fog, and new low-power networked sensor systems are under development to enable detection of smaller aircraft. With regard to airborne-based surveillance, advances in Detect-and-Avoid (DAA) technology will enable vehicles to automatically detect and track other aircraft, 
predict conflicts, and take corrective action as needed. Airborne and ground surveillance technologies could exchange information to validate conflicts. Air-based and ground-based surveillance target correlation may also assist in cybersecurity spoofing detection.

\section{Scope}

This section describes the scope of UAM airspace integration with regard to missions that could be performed, the aircraft that could utilized, the airspace where UAM flights could occur, and the hazards and risks involved in UAM operations. Other aspects of UAM, such as vehicle development, battery technology research, ground infrastructure construction, and legal considerations, are also important but outside of the scope of this paper.

\section{A. Mission Scope}

There is a desire by the UAM community to conduct a wide range of missions. The following is a partial list of potential operations that will be considered during NASA's development of UAM airspace integration concepts:

- On-demand air taxi operations moving people between fixed or ad hoc locations

- Air cargo operations moving goods between warehouses and stores

- Regularly scheduled "air metro" operations transporting passengers between a set of fixed locations

- Emergency medical evacuations, rescue operations, and humanitarian missions

- Law enforcement operations

- News gathering

- Weather monitoring

- Ground traffic assessment

\section{B. Aircraft Scope}

The aircraft concepts that have been proposed for UAM operations are diverse. Unlike conventional takeoff and landing transport aircraft, which generally have similar "tube-and-wing" configurations with wing-mounted engines, a wide range of different configurations have been proposed for UAM aircraft [26, 27, 28, 29, 30,31], and it is unclear which type(s) may end up being the preferred configuration(s). Furthermore, it is possible that different configurations will be better-suited for particular types of missions and metropolitan areas than others, which may result in different configurations being preferred in different settings.

The diversity of configuration types being explored at the present time echoes in many ways past research into VTOL aircraft. Researchers have previously experimented with a multitude of VTOL aircraft designs with varying degrees of success. Many of these past designs are documented in the V/STOL Aircraft and Propulsion Concepts "wheel" [32], which is often called the "Wheel of Misfortune" because only a few configurations resulted in practical aircraft. However, with the maturation of electric propulsion technologies, many of these previously ill-fated concepts may now be made practical due to the drastically different characteristics provided by electric propulsion. Specifically, electric propulsion can be distributed over the airframe with relatively few penalties due to the scale-invariance of electric motors and the ease of running wire around the airframe [11]. "Distributed electric propulsion" may enable aircraft that are more efficient, lower cost, faster, fault-tolerant, and less noisy than typical rotorcraft.

The many different aircraft configurations being proposed for UAM include separate lift and cruise propulsion [26, 27], (multi-) tiltrotors [28], (multi-) tiltwings [29], and multi-rotors [30, 31]. Each configuration has unique characteristics that will result in aircraft of varying performance. For example, some designs (e.g., tiltwings) are likely to be unable to hover for extended periods of time but may be capable of cruising at higher speeds. Other configurations (e.g., multi-rotors) will exhibit better hover performance but will be comparatively limited in terms of top speed and range. Another important distinguishing factor is how the aircraft transitions from vertical to horizontal flight (and back). Aircraft concepts like tiltwings and tiltrotors that require articulation-variable-geometry structure allowing reconfiguration of an aircraft for different flight regimes, such as wing-borne and powered-lift flight—will need non-negligible time, altitude, and distance to transition. By comparison, separate lift and cruise concepts and other concepts can perform more seamless transitions between vertical and horizontal flight.

UAM airspace integration concepts must take into account the wide range of performance that can be achieved by the diverse range of potential UAM aircraft types. More specifically, cruise speeds for these aircraft may range from approximately 70 knots up to 200 knots or more. In addition, hover capabilities may vary from on the order of ninety seconds to multiple tens of minutes. Transition times between vertical and horizontal flight may vary from near-zero to on the order of 30 seconds. These variations in performance will have significant implications on airspace design 
and operating procedures for airspace integration, such as holding patterns near vertiports, right-of-way rules, and/or constraints on vertiport access to vehicle classes with compatible operating envelopes.

\section{Airspace Scope}

By definition, the scope of UAM operations is within a metropolitan area. Class A airspace (i.e., between altitude $18,000 \mathrm{ft}$ and $60,000 \mathrm{ft} \mathrm{MSL}$ ) is out of scope for UAM airspace integration concepts because UAM aircraft are not expected to operate at such high altitudes. For metropolitan areas that are located further away from airports, UAM operations would have their origin, their destination, or both, be in Class E or Class G airspace. Although Class E airspace is controlled airspace, aircraft operating under VFR in Class E airspace are not required to be in contact with ATC. This is also true of flights in Class G, which is uncontrolled airspace.

On the other hand, for metropolitan areas that are located near airports, UAM operations may be conducted entirely within Class B airspace around major airports, Class C airspace around medium-sized airports, or Class D airspace around smaller airports. For example, downtown Dallas lies entirely within Class B airspace due to its proximity to Dallas-Fort Worth airport. The extent to which UAM operations would be allowed to occur in these classes of airspace would depend on the departure location, arrival location, and/or constraining factors (e.g., noise restrictions and obstacles). Under present-day rules, UAM flights would be required to communicate with ATC prior to entering Class $\mathrm{B}, \mathrm{C}$, or D airspace.

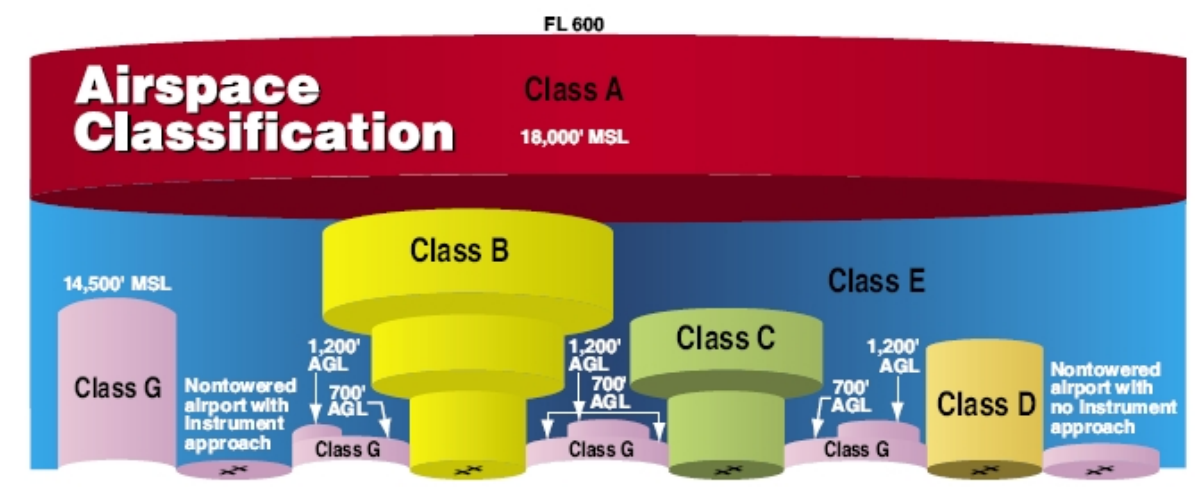

Fig. 2 Airspace classification.

\section{Hazard and Risk Scope}

Safety is paramount in conducting air traffic operations, and UAM is no exception. This section provides a highlevel overview of the scope of UAM hazards and risks that must be assessed for safe airspace integration of UAM. The terms "hazard" [33] and "risk" [34, 35, 36] are defined as follows for this paper:

- Hazard: A condition, event, object, or circumstance that could lead to or contribute to an unplanned or undesired event or state. The unplanned or undesired event/state may be the result of a single hazard but more often is the result of a series or combination of hazards interacting in a probabilistic manner.

- Risk: The combination of 1) the likelihood of a hazard/unplanned/undesired event or state occurring, 2) the amount of exposure to the hazard or duration of the event or state, 3) the severity of the hazard/event/state, and 4) the impacts or consequences of the hazard/event/state.

Table 1 is a sample set of the potential hazards that exist within and across the various UAM domains. Although some hazards may have few downstream consequences (e.g., a passenger canceling their flight), many hazards may have cascading effects that interact with other hazards. For example, a UAM vehicle requiring an emergency landing will need to have priority handling, which can disrupt the operations of other vehicles in the vicinity.

Once the list of hazards and component failure initiators has been identified, the next step is calculating risk to estimate each initiator's failure modes and the corresponding likelihood (i.e., probability) of occurrence. These failure modes will be the basis for the development of failure scenarios, which are defined through a sequence of failure events. Eventually, multiple end states (i.e., consequences) will be defined and quantified probabilistically with associated uncertainties that reflect lack of information. 
Table 1 Examples of UAM Domains and Potential Hazards

\begin{tabular}{|c|c|}
\hline UAM Domains & Potential Hazards \\
\hline Vehicle, equipage, systems* & $\begin{array}{ll}\text { - } & \text { Loss of electrical power to control systems } \\
\text { - } & \text { Failure or spoofing of GPS/Receiver Autonomous Integrity Monitoring }\end{array}$ \\
\hline Vehicle servicing and maintenance & - Unavailability of necessary replacement part \\
\hline $\begin{array}{l}\text { Communications [voice, datalink, } \\
\text { Command-and-Control (C2) link] }\end{array}$ & $\begin{array}{ll}\text { - } & \text { C2 link lost } \\
\text { - } & \text { Degraded Quality of Service (QoS) for critical control commands }\end{array}$ \\
\hline $\begin{array}{l}\text { Aerial operations, flight } \\
\text { procedures, flight management }\end{array}$ & $\begin{array}{ll}\text { - } & \text { Vehicle upset attitude } \\
\text { - } & \text { Vehicle fly-away } \\
\end{array}$ \\
\hline $\begin{array}{l}\text { Routing, airspace, air traffic } \\
\text { management }\end{array}$ & $\begin{array}{ll}\text { - } & \text { UAM route conflicts with existing air traffic } \\
\text { - } & \text { Loss of safety-critical functions on ground station }\end{array}$ \\
\hline $\begin{array}{l}\text { External environment (weather, } \\
\text { obstacles, aerial traffic, birds) }\end{array}$ & $\begin{array}{l}\text { - } \quad \text { Convective weather (hail, severe downdrafts) } \\
\text { - } \quad \text { Buildings, power lines, airborne vehicles }\end{array}$ \\
\hline Pilots (on-board and remote) & $\begin{array}{l}\text { - Inadequate pilot training for maintaining safety margins } \\
\text { - Loss of pilot situational awareness } \\
\end{array}$ \\
\hline $\begin{array}{l}\text { Dispatch, control center, } \\
\text { "emergency pilots"** }\end{array}$ & $\begin{array}{ll}\text { - } & \text { Dispatch understaffed, flight planning delayed } \\
\text { - } & \text { Loss or degradation of ground control station capability (e.g., displays) }\end{array}$ \\
\hline $\begin{array}{l}\text { Ground-based operations and } \\
\text { infrastructure }\end{array}$ & $\begin{array}{l}\text { - Lack of vertiport availability (occupied, damaged, closed to traffic) } \\
\text { - } \quad \text { Inadequate ground crew training for maintaining safety margins }\end{array}$ \\
\hline Passengers & $\begin{array}{ll}\text { - } & \text { Passenger interference with pilot/vehicle operations } \\
\text { - } & \text { Passenger illness during flight } \\
\end{array}$ \\
\hline Cybersecurity (including V\&V) & - Inadequate authentication of C2 link (undetected hijacking of C2 link) \\
\hline
\end{tabular}

\section{High-Level Concept Descriptions}

This section describes at a high level NASA's initial airspace integration concepts for both emergent and early expanded UAM operations. The former is expected to be characterized by low-tempo, low-density operations that are similar to VFR flights along a small set of fixed routes between a few takeoff and landing areas. The latter is envisioned to involve higher-tempo, higher-density operations in a small network of vertiports feeding a common hub location and managed by UAM operator and third-party services. Beyond the scope of this paper, NASA is also planning to develop a concept for high-tempo, high-density mature UAM operations in a network with multiple hub locations, potentially with orders-of-magnitude more vehicles and operations in an area than are currently supported in the NAS.

\section{A. Emergent Operations}

It is expected that the earliest UAM operations in the future will be to demonstrate the potential benefits of UAM to the general public and metropolitan areas. These initial UAM flights will be used to gather preliminary feedback from surrounding communities (e.g., on noise) and gain support for initial commercial operations. It is anticipated that these flights will operate under VFR with human pilots on board at all times and will be subject to the same equipage requirements and airspace rules and regulations as existing VFR flights. Furthermore, it is expected these initial UAM flights would require ATC to provide air traffic services and management similar to (or the same as) VFR flights in current operations. For instance, these initial UAM flights will be required to communicate with ATC to conduct operations into and out of Class B, Class C, and Class D controlled airspace in the vicinity of airports (Section IV.C). The constraints on ATC workload is a limiting factor on the tempo and density of emergent UAM operations.

Passenger-carrying UAM flights using conventional helicopters operating under VFR with pilots on board already occur in the airspace. However, the range of other types of UAM aircraft that will also be allowed to conduct UAM operations will depend on certification. While many UAM aircraft manufacturers are designing vehicles to have a pilot on board the aircraft, others are designing vehicles to be remotely piloted and supervised by humans on the ground and/or autonomous systems in the air and/or on the ground. The authorization to conduct UAM flightspassenger-carrying or otherwise - without pilots on board the aircraft is dependent on certification of these vehicles and authorization of these operations, which has not yet occurred. It is also dependent on the certification of pilots and support systems on the ground, which has also not yet occurred. As such, certification is another limiting factor on the tempo and density of emergent UAM operations. 
Besides UAM aircraft manufacturers needing to develop vehicles and gain certification, ground infrastructure developers also need to build the vertiports and the re-fueling infrastructure required to support UAM operations. It is anticipated that the number of vertiports, the number of vertipads, and the speed of re-fueling UAM aircraft will be limited in the near term. Ground infrastructure development is another constraining factor on the tempo and density of emergent UAM operations.

With regard to the routes that UAM will traverse between two vertiports, a natural starting point for emergent UAM operations is to fly along defined helicopter routes. The FAA has published helicopter route charts for eight metropolitan areas: Baltimore-Washington, Boston, Chicago, Dallas-Fort Worth (Figure 3), Detroit, Houston, Los Angeles, and the U.S. Gulf Coast [37]. These helicopter routes tend to overlay highways and freeways on the ground to mitigate societal concerns like noise and also to provide visual reference for pilots while in the air.

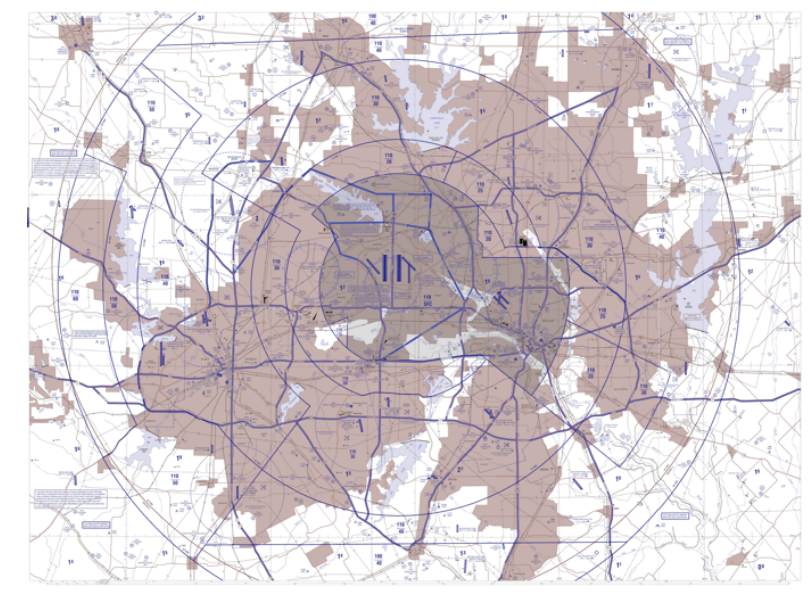

Fig. 3 Helicopter routes in the Dallas-Fort Worth area.

Another possibility for emergent UAM operations are corridors similar to the Los Angeles Special Flight Rules Area (SFRA) as specified in the Code of Federal Regulations (CFR) 14 CFR § 93.95. The Los Angeles SFRA (Figure 4) consists of two routes that are both on the Santa Monica Very High Frequency Omni-Directional Range (VOR) $132^{\circ}$ radial. Flights traveling southeast operate at $3500 \mathrm{ft}$ MSL, and flights traveling northwest operate at $4500 \mathrm{ft}$ MSL. Aircraft in this corridor are not required to communicate with ATC even though they are in Class B airspace. The current lack of ATM services like sequencing, scheduling, and spacing in corridors like the Los Angeles SFRA is a limiting factor on the tempo and density of UAM operations that they can support.

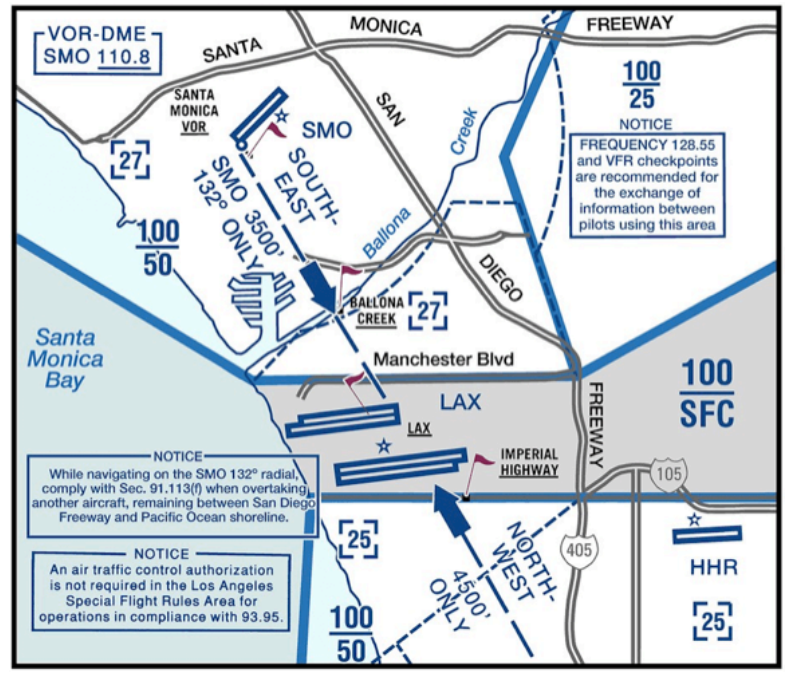

Fig. 4 Los Angeles Special Flight Rules Area. 
Whether along helicopter routes, inside of corridors, or other airspace constructs, it is anticipated that UAM flights could operate in the vicinity of all other types of aircraft: both manned and unmanned aircraft operating under either IFR or VFR. It is not expected that UAM operations would be segregated from other air traffic operations. This means that UAM aircraft and systems must safely interoperate with other aircraft and their systems on the ground and in the air. This includes large UAS, their DAA systems, and their well clear separation standard [38].

\section{B. Early Expanded Operations}

The NAS currently has three fundamental bottlenecks during clear weather operations: gates, runways, and ATC workload. While UAM is unlikely to use conventional gates and runways, ATC workload may limit UAM if not properly designed. As such, developing the concepts, technologies, and procedures that will enable UAM to be integrated into the airspace system and managed without tactical intervention from ATC represents a significant portion of the effort toward achieving higher-density, higher-tempo UAM operations.

A service-oriented architecture and paradigm like UTM [2] is one possibility for future UAM operations. In this case, many services could be offered by UAM operators and third parties. While some services may be offered by a single provider similar to a utility company, other services may be offered competitively by multiple providers similar to mobile phone service companies. In this paradigm, the role of the Air Navigation Service Provider (ANSP), such as the FAA, is to set constraints and directives but not to actively manage every UAM aircraft and trajectory change by clearances. Rather, the ANSP will provide the information that UAM operators need to safely and efficiently manage their flights.

UAM operators will need to interact with each other and service providers for collision avoidance, tactical conflict detection and resolution, sequencing, scheduling, and spacing into and out of vertiports, as well as overall network demand-capacity management. This will require information sharing and systems performing to defined performance standards. Given that UAM operators and service providers will be responsible for separation from other aircraft and the various obstacles in metropolitan areas (e.g., buildings, power lines, trees), they will need to conduct surveillance of the vehicles, maintain obstacle databases, and detect and mitigate contingencies (e.g., lost communication, equipment malfunction). UAM operators will be responsible for alerting as early as possible other operators who may be impacted by a contingency action.

The ANSP will provide constraints and directives to ensure the safe integration of UAM operations with the other aircraft operations in the NAS. UAM operators and service providers will work within these constraints and directives to safely and efficiently conduct flights, which are expected to primarily be done using eVTOL aircraft potentially traveling along routes defined ad hoc. Together, this is expected to enable significantly higher-tempo and higherdensity operations than are currently supported in the NAS.

\section{Future Work}

NASA's UAM airspace integration effort will focus on enabling early entrants in the airspace and exploring possibilities for the services, procedures, and tools necessary to support high-tempo, high-density mature operations. Given the early stage of development for UAM airspace integration at the present time, NASA has embraced an agile "Build, Explore, and Learn" collaborative approach. With regard to the learning aspect, NASA plans to share information and ideas with the UAM community on a regular basis. NASA is also looking to learn from the UAM community and the broader aviation community regarding UAM airspace integration.

NASA will engage with partners and stakeholders to develop and refine the initial airspace integration concepts for both emergent and early expanded UAM operations described in this paper, as well as a concept for high-tempo, high-density mature UAM operations. NASA also plans to engage with partners and stakeholders to collaborate on the research and development of technologies and standards for UAM operations; these collaborative activities could include fast-time simulations, human-in-the-loop evaluations, and live flight demonstrations.

\section{A. UAM Air Traffic Management Research}

The technologies needed to integrate UAM operations into the NAS span the ATM spectrum, though with various new considerations unique to UAM. NASA plans to investigate possibilities for the services, procedures, and tools that are needed to address UAM airspace integration barriers. This will include leveraging knowledge gained through prior research efforts to develop concepts, technologies, and procedures for traditional aviation and new entrants like UAS. Novel innovations in UAM airspace integration research may also be transferred to improve air traffic operations for existing airspace users.

NASA's Air Traffic Management-eXploration (ATM-X) project [15] is currently investigating one framework for managing UAM operations in which strategic management components like congestion management (Section VI.A.1) 
and vertiport network scheduling (Section VI.A.2) are integrated with tactical management components like en-route separation and departure and arrival management at vertiports (Section VI.A.3). These components will need to be interoperable (Section VI.A.4) and resilient to disruptions (Section VI.A.5) and contingencies (Section VI.A.6). This section describes these components and considerations for UAM airspace integration. It is expected that other frameworks for managing UAM operations will be investigated as NASA learns with and collaborates with the UAM community and the broader aviation community.

\section{Congestion Management}

Dense areas of traffic constrain the operational flexibility to respond to uncertainties and disruptions, which can lead to flight delays, extended flight times, and diversions of flights to alternate destinations. Congestion management can enable increased predictability and increased operational flexibility to respond to uncertainties. It could include methods to manage the scheduling and routing of traffic flows through an airspace region and/or to limit the number of vehicles. It is expected that the management of aircraft taking off from and landing at vertiports and vertipads located in close proximity to each other will be a central part of UAM congestion management. Research could include defining the routes and developing the schedules for departure and arrival at vertiports and vertipads.

\section{Scheduling}

Due to limited energy reserves, UAM aircraft must have assurances prior to takeoff that their destination landing site will be available when they arrive. The tight coupling between arrivals and departures across the vertiports in a UAM network points to the possible need for continuous network-wide scheduling as a first-order control method for real-time, on-demand resource management. The capability to schedule across a UAM network may also be needed for congestion management. Network-wide scheduling will also need to consider how individual vertiports conduct sequencing, scheduling, and spacing of UAM arrivals and departures. This could include the development of route structures between UAM vertiports (e.g., multiple paths for departures and/or arrivals) for safety, efficiency, capacity, and community acceptance (e.g., noise).

\section{Separation}

Although strategic congestion management and vertiport network scheduling are envisioned to be an important component for enabling safe, efficient, and equitable UAM operations, they may not be sufficient due to uncertainty in UAM flight trajectories, environmental conditions (e.g., wind), and other factors. Tactical departure management, en-route separation management, and arrival management technologies may also be needed to mitigate the effects of uncertainty. These technologies will need to take into account the unique considerations of UAM, such as ground infrastructure capabilities (e.g., to refuel UAM aircraft) and vehicle constraints (e.g., remaining battery charge), that are more significant for UAM than for traditional aviation.

The separation standard that these separation technologies will work to maintain is an area of research, and it will be driven by a target level of safety that needs to be determined. One approach for preserving safe separation and achieving the target level of safety that could be investigated is having multiple systems operating on different time frames working together on encounter situations. The extent to which these systems are centralized, the level of autonomy, and the role of humans are all aspects of separation for UAM that need to be explored.

\section{Interoperability}

The safe airspace integration of UAM vehicles and systems will require that they be interoperable with each other, as well as the vehicles and systems of existing airspace users. More generally, interoperability is essential for a serviceoriented data exchange architecture and the harmonization of different technologies. At a minimum, standards must be developed for the data exchange architecture and CNS services. In addition, it may be necessary to develop protocols to ensure the integrity, timeliness, and consistent understanding of the information being exchanged by different vehicles and systems.

Prior research on the interoperability of the strategic conflict detection and resolution algorithm and the tactical conflict detection and resolution algorithm of a separation management system highlighted the criticality of data integrity, communication timeliness, and consistent understanding of information by different systems [39]. In addition to ensuring effective communication, it is essential to develop methods and procedures for the vehicles and systems of UAM and other airspace users to operate in a compatible way with each other. For example, in the case of large UAS, the interoperability of DAA systems on board UAS and TCAS systems on board manned aircraft was identified as a research gap [40] and was addressed in a systematic way using mathematical expressions derived from analysis of aircraft encounters [41]. Similar types of research may need to be performed to ensure the safe integration of UAM operations into the airspace system. 


\section{Disruption Management}

In addition to congestion management, vertiport network scheduling, and separation, it is also envisioned that disruption management will be an important part of managing UAM operations. For example, weather is one type of disruption that is expected to be a major factor in UAM operations, just as it is for traditional manned aviation and UAS. Disruption management includes identifying and avoiding potentially hazardous areas of the airspace before takeoff and while in the air. It also includes managing UAM aircraft in contingency situations like emergency landings. In the presence of disruptive events and after determining the cause of the disruption, a disruption management service could provide disruption information, modified flight plans, expected times at critical reference locations along the flight route, and/or mitigation strategies as requested by UAM operators.

\section{Contingency Response Management}

Autonomous systems have historically had trouble with unexpected contingency situations. Carlos Ghosn, chairman and then-CEO of Nissan said, "No matter how powerful [the artificial intelligence] is, we always find a case where the car will be stuck" [42]. One approach being pursued by Nissan is to use contingency response centers where human contingency response managers will be on call to take care of situations that technology cannot handle.

The role of contingency response managers in UAM could naturally evolve from the role of dispatchers in the current ATM system. In present-day commercial operations for traditional aviation, the dispatcher is responsible for safety of flight in conjunction with the captain of the aircraft. Initially, a contingency response manager in UAM could be expected to step in to help pilots, similar to how dispatchers provide assistance to pilots when needed in current operations. As autonomous systems become more responsible for piloting, the contingency response manager could become more responsible for handling situations that are beyond the capability of the autonomous system. The extent to which this human-centric paradigm for contingency response management is applicable to the different stages of UAM operational maturity is a research area to be explored.

\section{Concept for Mature UAM Operations}

NASA will engage with partners and stakeholders to develop a concept for high-tempo, high-density mature UAM operations with potentially orders-of-magnitude more vehicles and operations in an area than are currently supported in the NAS. Achieving this could provide a viable alternative to ground transportation in metropolitan areas. It is anticipated that the progression toward mature UAM operations will begin with low-risk, low-tempo, low-density UAM operations. More complex operations will be added incrementally while ensuring overall system safety. At each stage of UAM operational maturity, new lessons will be learned and applied.

\section{B. UAM Hazard and Risk Research}

UAM-related hazards and risks will be addressed across several NASA projects. For example, under NASA's System Wide Safety (SWS) project, hazards and risks associated with UAM validation and verification tools and methods will be addressed through the development of assurance tools and methods to provide guaranteed behavior in unpredictable aviation contexts. This work will demonstrate the effectiveness of assurance tools and methods for candidate autonomous and complex non-deterministic UAM systems. With regard to the system-wide safety of emergent systems, initial safety-critical risks to be addressed include:

- Flight outside of approved airspace

- Unsafe proximity to people/property

- Critical system failure (e.g., loss of C2 link, loss or degraded GPS, loss of power, and engine failure)

- Loss of control (i.e., outside the envelope or flight control system failure)

- Cybersecurity-related risks

Furthermore, a comprehensive list of hazards across the UAM domain areas will be identified, and the associated risk level of each hazard will be evaluated. Mitigations for those hazards will be developed in collaboration with industry partners.

\section{Concluding Remarks}

This paper described at a high level NASA's initial airspace integration concepts for both emergent and early expanded UAM operations. The scope of the work was defined in terms of missions, aircraft, airspace, and hazards. In addition, the considerations for the data exchange architecture and communication, navigation, and surveillance 
requirements were also discussed. This paper also detailed the goals, principles, barriers, and benefits of UAM, as well as the competing considerations that need to be taken into account and balanced.

NASA has embraced an agile "Build, Explore, and Learn" collaborative approach for UAM airspace integration and will engage with partners and stakeholders to work together to: 1) develop and refine the initial concepts for both emergent and early expanded UAM operations, 2) develop a concept for high-tempo, high-density mature UAM operations with more vehicles in an area than are currently supported in the NAS, and 3) research and develop the technologies and procedures that will inform and enable these concepts. These efforts could include fast-time simulations, human-in-the-loop evaluations, and live flight demonstrations.

\section{References}

[1] Moore, M. D., Goodrich, K., Viken, J., Smith, J., Fredericks, B., Trani, T., Barraclough, J., German, B., and Patterson, M., "High-Speed Mobility through On-Demand Aviation," 2013 Aviation Technology, Integration, and Operations Conference, AIAA AVIATION Forum, AIAA 2013-4373, Aug. 2013. doi: $10.2514 / 6.2013-4373$

[2] Kopardekar, P., Rios, J., Prevot, T., Johnson, M., Jung, J., and Robinson, J. E., "UAS Traffic Management (UTM) Concept of Operations to Safely Enable Low Altitude Flight Operations," 16th AIAA Aviation Technology, Integration, and Operations Conference, AIAA AVIATION Forum, AIAA 2016-3292, Jun. 2016. doi: $10.2514 / 6.2016-3292$

[3] Antcliff, K., Moore, M., and Goodrich, K., "Silicon Valley as an Early Adopter for On-Demand Civil VTOL Operations," 16th AIAA Aviation Technology, Integration, and Operations Conference, AIAA AVIATION Forum, AIAA 2016-3466, Jun. 2016.

doi: $10.2514 / 6.2016-3466$

[4] Holden, J., and Goel, N., "Uber Elevate: Fast-Forwarding to a Future of On-Demand Urban Air Transportation," Uber Inc., San Francisco, CA, 2016.

[5] Lewis, J. and Magalhaes, L., "Here Come Helicopters on Demand," The Wall Street Journal, Jun. $20,2017$. https://www.wsj.com/articles/here-come-helicopters-on-demand-1498010700

[6] Sorkin, A. R., “Larry Page’s Flying Taxis, Now Exiting Stealth Mode,” The New York Times, Mar. 12, 2018. https://www.nytimes.com/2018/03/12/business/dealbook/flying-taxis-larry-page.html

[7] Moon, M., "Dubai tests a passenger drone for its flying taxi service," Engadget, Sep. 26, 2017. https://www.engadget.com/2017/09/26/dubai-volocopter-passenger-drone-test/

[8] Holmes, B. J., and Parker, Roger A., “A Vision and Opportunity for Transformation of On-Demand Air Mobility,” 16th AIAA Aviation Technology, Integration, and Operations Conference, AIAA AVIATION Forum, AIAA 2016-3465, Jun. 2016. doi: $10.2514 / 6.2016-3465$

[9] Nneji, V. C., Stimpson, A., Cummings, M., and Goodrich, K. H., "Exploring Concepts of Operations for On-Demand Passenger Air Transportation," 17th AIAA Aviation Technology, Integration, and Operations Conference, AIAA AVIATION Forum, AIAA 2017-3085, Jun. 2017. doi: $10.2514 / 6.2017-3085$

[10] Narkus-Kramer, M., "On-Demand Mobility (ODM): A Discussion of Concepts and Required Research,” 2013 Aviation Technology, Integration, and Operations Conference, AIAA AVIATION Forum, AIAA 2013-4294, Aug. 2013. doi: $10.2514 / 6.2013-4294$

[11] Moore, M. D. and Fredericks, B., "Misconceptions of Electric Aircraft and their Emerging Aviation Markets," 52nd Aerospace Sciences Meeting, AIAA SciTech Forum, AIAA 2014-0535, Jan. 2014. doi: $10.2514 / 6.2014-0535$

[12] Garrow, L. A., Ilbeigi, M., and Chen, Z., "Forecasting Demand for On Demand Mobility," 17th AIAA Aviation Technology, Integration, and Operations Conference, AIAA AVIATION Forum, AIAA 2017-3280, Jun. 2017. doi: $10.2514 / 6.2017-3280$ 
[13] Smith, J. C., Viken, J. K., Guerreiro, N. M., Dollyhigh, S. M., Fenbert, J. W., Hartman, C. L., Kwa, T., Moore, M. D., "Projected Demand and Potential Impacts to the National Airspace System of Autonomous, Electric, On-Demand Small Aircraft," 12th AIAA Aviation Technology, Integration, and Operations (ATIO) Conference and 14th AIAA/ISSMO Multidisciplinary Analysis and Optimization Conference, Sep. 2012. doi: $10.2514 / 6.2012-5595$

[14] Mueller, E., Kopardekar, P., and Goodrich, K., "Enabling Airspace Integration for High-Density Mobility Operations," 17th AIAA Aviation Technology, Integration, and Operations Conference, AIAA AVIATION Forum, AIAA 2017-3086, Jun. 2017. doi: $10.2514 / 6.2017-3086$

[15] Chan, W. N., Barmore, B. E., Kibler, J., Lee, P., O’Connor, N., Palopo, K., Thipphavong, D., and Zelinski, S., “Overview of NASA's Air Traffic Management-eXploration (ATM-X) Project," 18th AIAA Aviation Technology, Integration, and Operations Conference, AIAA AVIATION Forum, Jun. 2018 (in print).

[16] Harrison, S., "From the Archives: Los Angeles Airways helicopter overturns," The Los Angeles Times, Mar. $10,2017$. http://www.latimes.com/visuals/photography/la-me-fw-archives-airways-helicopter-overturn-20170221-story.html

[17] Witken, R., "New York Airways Acts To File for Bankruptcy,” The New York Times, May 16, 1979. https://www.nytimes.com/1979/05/16/archives/new-york-airways-acts-to-file-for-bankruptcy-suing-sikorsky.html

[18] Barron, J., “The Citizens of East Hampton v. Its Airport,” The New York Times, Jul. 4, 2017. https://www.nytimes.com/2017/07/04/nyregion/east-hampton-airport-noise-regulations.html

[19] Wingfield, N., and Scott, M., "In Major Step for Drone Delivery, Amazon Flies Package to Customer in England," The New York Times, Dec. 14, 2016. https://www.nytimes.com/2016/12/14/technology/amazon-drone-england-delivery.html

[20] Overly, S., "In Rwanda, lifesaving blood now drops from drones,” The Washington Post, Oct 13, 2016. https://www.washingtonpost.com/news/innovations/wp/2016/10/13/in-rwanda-life-saving-blood-now-drops-from-the-sky/

[21] Rios, J., Mulfinger, D., Homola, J., Venkatesan, P., "NASA UAS Traffic Management National Campaign-Operations Across Six UAS Test Sites," IEEE-DASC 2016, Sep. 2016. doi: 10.1109/DASC.2016.7778080

[22] Aweiss, A. S., Owens, B. D., Rios, J. L., Homola, J., Mohlenbrink, C. P., "Unmanned Aircraft Systems (UAS) Traffic Management (UTM) National Campaign II”, 2018 AIAA Information Systems-AIAA Infotech @ Aerospace, AIAA SciTech Forum, AIAA 2018-1727, Jan. 2018. doi: $10.2514 / 6.2018-1727$

[23] Federal Highway Administration, "Urban Congestion Report October - December 2016," https://ops.fhwa.dot.gov/perf_measurement/ucr/reports/52urbanareas/fy2017_q1.pdf

[24] RTCA, SC-223 Internet Protocol Suite (IPS) and AeroMACS.

[25] "Manual on the Aeronautical Telecommunication Network (ATN) using Internet Protocol Suite (IPS) Standards and Protocol," ICAO, Working Group Internetwork, Internet Protocol Suite-Mobility, Montreal, Quebec, Canada, 2015.

[26] "Press - Cora,” Apr. 16, 2018. https://cora.aero/press/

[27] “eVTOL - Aurora Flight Sciences,” Apr. 16, 2018. http://www.aurora.aero/evtol/

[28] “Joby Aviation,” Apr. 16, 2018. http://www.jobyaviation.com/

[29] “A³ Vahana,” Apr. 16, 2018. https://www.airbus-sv.com/projects/1

[30] "Volocopter - Product," Apr. 16, 2018. https://www.volocopter.com/en/product/

[31] “SureFly | Workhorse,” Apr. 16, 2018. http://workhorse.com/surefly

[32] Hirschberg, M. J., "V/STOL: The First Half-Century,” Vertiflite, Vol. 43, No. 2, Mar./Apr. 1997.

[33] "Risk Management Handbook," U.S. Department of Transportation, Federal Aviation Administration, Flight Standards Service, FAA-H-8083-2, Washington, D.C., 2009. 
[34] Leveson, N. G., Safeware: System Safety and Computers, ACM, New York, NY, Apr. 1995.

[35] Glenn, K., Jansen, M., and Gutierrez O., "Quantitative Risk Assessment NASA OH3/Assessments, Cost Estimates \& Schedules," Aug. 2015.

[36] Moses, K. D., and Malone, R. W., "Development of Risk Assessment Matrix for NASA Engineering and Safety Center," Risk Analysis: The Profession and the Future, Dec. 2004.

[37] "VFR Raster Charts," Federal Aviation Administration, Jan. 31, 2017. https://www.faa.gov/air_traffic/flight_info/aeronav/digital_products/vfr/

[38] "Minimum Operational Performance Standards (MOPS) for Detect and Avoid (DAA) Systems,” RTCA, DO-365, Washington, D.C., May 2017.

[39] Thipphavong, D., "Exploratory Study of Interoperability Between Tactical and Strategic Separation Assurance Functions," 2013 AIAA Aviation Technology, Integration, and Operations Conference, AIAA AVIATION Forum, AIAA 2013-4333, Aug. 2013.

doi: $10.2514 / 6.2013-4333$

[40] Londner, E. H., "Interoperability of Horizontal and Vertical Resolution Advisories," 11th USA/Europe Air Traffic Management Research and Development Seminar, Paper 427, Jun. 2015.

[41] Thipphavong, D., Cone, A., Lee, S. M., "Ensuring Interoperability between UAS Detect-and-Avoid and Manned Aircraft Collision Avoidance," 12th USA/Europe Air Traffic Management Research and Development Seminar, Paper 54, Jun. 2017.

[42] Lawler, R. “Nissan's SAM uses humans as a backup for self-driving tech,” Engadget, Jan. 5, 2017. https://www.engadget.com/2017/01/05/nissans-sam-uses-humans-as-a-backup-for-self-driving-tech/ 